Proyecciones Journal of Mathematics Vol. 31, No 1, pp. 11-24, March 2012. Universidad Católica del Norte Antofagasta - Chile

\title{
On the Gauss-Newton method for solving equations
}

\author{
IOANNIS K. ARGYROS \\ CAMERON UNIVERSITY, U.S.A. \\ and \\ SAÏD HILOUT \\ POITIERS UNIVERSITY, FRANCE \\ Received : January 2011. Accepted : October 2011
}

\begin{abstract}
We use a combination of the center-Lipschitz condition with the Lipschitz condition condition on the Fréchet-derivative of the operator involved to provide a semilocal convergence analysis of the GaussNewton method to a solution of an equation. Using more precise estimates on the distances involved, under weaker hypotheses, and under the same computational cost, we provide an analysis of the GaussNewton method with the following advantages over the corresponding results in [8]: larger convergence domain; finer error estimates on the distances involved, and an at least as precise information on the location of the solution
\end{abstract}

AMS Subject Classification. 65F20, 65G99, 65H10, 49M15.

Key Words. Gauss-Newton method, semilocal convergence, Fréchetderivative, Lipschitz/center-Lipschitz condition, convergence domain. 


\section{Introduction}

In this study we are concerned with the problem of approximating a solution $x^{\star}$ of equation

$$
F^{\prime}(x)^{T} F(x)=0,
$$

where $F$ is a Fréchet-differentiable operator defined on $X=\mathbb{R}^{n}$, with values on $Y=\mathbb{R}^{m}(m \geq n)$.

A large number of problems in applied mathematics and also in engineering are solved by finding the solutions of certain equations. For example, dynamic systems are mathematically modeled by difference or differential equations, and their solutions usually represent the states of the systems. For the sake of simplicity, assume that a time-invariant system is driven by the equation $\dot{x}=T(x)$, for some suitable operator $T$, where $x$ is the state. Then the equilibrium states are determined by solving equation (1.1). Similar equations are used in the case of discrete systems. The unknowns of engineering equations can be functions (difference, differential, and integral equations), vectors (systems of linear or nonlinear algebraic equations), or real or complex numbers (single algebraic equations with single unknowns). Except in special cases, the most commonly used solution methods are iterative-when starting from one or several initial approximations a sequence is constructed that converges to a solution of the equation. Iteration methods are also applied for solving optimization problems. In such cases, the iteration sequences converge to an optimal solution of the problem at hand. Since all of these methods have the same recursive structure, they can be introduced and discussed in a general framework.

We are seeking least-square solutions of (1.1). That is we solve the minimization problem:

$$
\min _{x \in X} \frac{1}{2} F(x)^{T} F(x) .
$$

We use the famous Gauss-Newton method

$$
\left(1 x_{k}\right)_{+1}=x_{k}-\left(F^{\prime}\left(x_{k}\right)^{T} F^{\prime}\left(x_{k}\right)\right)^{-1} F^{\prime}\left(x_{k}\right)^{T} F\left(x_{k}\right) \quad\left(x_{0} \in \mathbb{R}^{n}\right), \quad(k \geq 0)
$$

to generate a sequence approximating a solution $x^{\star}$ of (1.2).

There is an extensive literature on the local as well as the semilocal convergence analysis of Newton-type methods under various conditions in 
the more general setting when $X$ and $Y$ are Banach spaces [1]-[10].

In particular, in the case of Gauss-Newton method (1.3), Li et al. provided a semilocal convergence analysis in [8] using the concept of the Lipschitz condition.

Recently, we have successfully used in [1]-[3] a combination of Lipschitz and center-Lipschitz conditions instead of only Lipschitz conditions as in to provide a finer local and semilocal convergence analysis for Newton-type methods, when $F$ is an isomorphism. The main idea is derived from the observation that more precise upper bounds on the norms $\left\|F^{\prime}(x)^{-1} F^{\prime}\left(x^{\star}\right)\right\|$ can be obtained if the needed center-Lipschitz condition is used:

$\left\|F^{\prime}\left(x^{\star}\right)^{-1}\left(F^{\prime}(x)-F^{\prime}\left(x^{\star}\right)\right)\right\| \leq L_{0} \quad\left\|x-x^{\star}\right\|$,

for all $x \in U\left(x^{\star}, r\right)=\left\{x \in X:\left\|x-x^{\star}\right\| \leq r\right\} \subseteq X, r>0, L_{0}>0$

instead of

$\left\|F^{\prime}\left(x^{\star}\right)^{-1}\left(F^{\prime}(x)-F^{\prime}(y)\right)\right\| \leq L\|x-y\|, \quad$ for all $x, y \in U\left(x^{\star}, r\right), L>0$

(which is commonly used in [4]-[10]).

It turns out that these ideas can be used to study the semilocal convergence of the Gauss-Newton method (1.3). In particular, we provide a semilocal convergence analysis with the following advantages over the work by Li et al. [8]:

1. Weaker sufficient convergence conditions;

2. Larger convergence domain;

3. Finer estimates on the distances involved, which implies that fewer iterations are needed to achieve a desired error tolerance.

4. An at least as precise information is provided on the uniqueness of the solution.

The above improvements are also obtained under the same computational cost since the computation of the Lipschitz condition with constant $\ell$ (see (1.4)) requires that of the center-Lipschitz condition with constant $\ell_{0}$ (see $(1.5))$. 
In particular, we assume there exist $\ell>0$ such that the Lipschitz condition

$$
\begin{aligned}
& \left\|F^{\prime}(x)-F^{\prime}(y)\right\| \leq \ell\|x-y\|, \\
& \text { holds for all } x, y \in D .
\end{aligned}
$$

In view (1.4), there exists $\ell_{0}>0$ such that center-Lipschitz condition

$$
\begin{aligned}
& \left\|F^{\prime}(x)-F^{\prime}\left(x_{0}\right)\right\| \leq \ell_{0} \quad\left\|x-x_{0}\right\|, \\
& \text { holds for all } x \in D
\end{aligned}
$$

Note that in [8], the same Lipschitz constant $\ell$ is used in (1.4) and (1.5).

However,

$$
a=\frac{\ell_{0}}{\ell} \leq 1
$$

holds in general, and $a$ can be arbitrarily small [1]-[3].

Let us provide a simple example where strict inequality holds in (1.6). Let $n=m=1, x_{0}=1$, and define function $F$ on $D=[\delta, 2-\delta], \delta \in\left[0, \frac{1}{2}\right)$ by

$$
F(x)=x^{3}-\delta
$$

Using (1.4)-(1.7), we obtain

$$
\ell_{0}(t)=3-\delta<2(2-\delta)=\ell \quad \text { for all } \delta \in\left[0, \frac{1}{2}\right) .
$$

Using weaker $(1.5)$ (if $\ell_{0}<\ell$ ) (which is actually needed in the computation of the norms

$$
\left\|\left(F^{\prime}(x)^{T} F^{\prime}(x)\right)^{T} F^{\prime}(x)^{T}\right\|\left\|F^{\prime}(x)-F^{\prime}\left(x_{0}\right)\right\|
$$

instead of the stronger hypotheses (1.4) used in [8], leads us to the advantages as stated in the abstract of this study of our approach over the corressponding ones in [8].

\section{Semilocal convergence analysis of the Gauss-Newton method (1.3)}

Let $\mathbb{R}^{m \times n}$ be the set of all $m \times n$ matrices, and $A^{+}$be the generalized inverse of $A \in \mathbb{R}^{m \times n}$. Then, when $m \geq n$, and $A$ is of full rank, we have $A^{+}=\left(A^{T} A\right)^{-1} A^{T}$. 
We need the lemmas: [8], [9], [10] Let $A, E \in \mathbb{R}^{m \times n}$. Assume $B=$ $A+E$, and $\left\|A^{+}\right\|\|E\|<1$. Then, the following hold:

$$
\operatorname{rank}(B) \geq \operatorname{rank}(A) .
$$

If $\operatorname{rank}(A)=n, m \geq n$, then we have $\operatorname{rank}(B)=n$. [8], [9], [10] Let $A, E \in \mathbb{R}^{m \times n}$. Assume $B=A+E$, and $\left\|A^{+}\right\|\|E\|<1$. Then, the following hold:

$$
\left\|B^{+}\right\| \leq \frac{\left\|A^{+}\right\|}{1-\left\|A^{+}\right\|\|E\|}
$$

provided that $\operatorname{rank}(B)=\operatorname{rank}(A)$.

Moreover,

$$
\left\|B^{+}-A^{+}\right\| \leq \frac{\sqrt{2}\left\|A^{+}\right\|^{2}\|E\|}{1-\left\|A^{+}\right\|\|E\|}
$$

provided that $\operatorname{rank}(A)=\operatorname{rank}(B)=\min \{m, n\}$. It is convenient for us to define for each fixed $a \in(0,1]$, functions $f_{a}$ and $g_{a}$ on $[0,1]$ by

$$
f_{a}(t)=\left(\begin{array}{l}
\left.\sqrt{2}\left(1+p_{1}\right)-(1+a p)(1+2 a p q)\right) t^{2}+ \\
1+2 \sqrt{2}+2 q(1+a p)+2 a p q) t-2 q,
\end{array}\right.
$$

and

$$
g_{a}(t)=(\sqrt{2}-a) t^{2}-(2 a+5 \sqrt{2}) t+3 \sqrt{2}-a,
$$

where,

$$
\begin{gathered}
q=\frac{2 a+5 \sqrt{2}-\sqrt{26+36 a \sqrt{2}}}{2(\sqrt{2}-a)}, \\
q=\frac{1}{1-q}
\end{gathered}
$$

and

$$
p_{1}=\frac{q^{2}}{1-q^{2}} .
$$

It is simple algebra to show $q$ is the small positive zero of function $g_{a}$ with $q \in(0,1)$. We also have $f_{a}(0)=-2 q<0$, and

$$
f_{a}(1)=\frac{g_{a}\left(q^{0}\right)}{\left(1-q^{0}\right)^{2}\left(1+q^{0}\right)} \geq 0, \quad \text { for all } \quad q^{0} \in[0, q] .
$$


It follows from the intermediate value theorem that there exists a maximal $h_{0} \in[0,1]$ such that

$$
f_{a}(t) \leq 0 \text { for all } t \in\left[0, h_{0}\right] .
$$

Define also $h_{1}, b$ and $c$ by

$$
\begin{gathered}
h_{1}=\frac{1-q}{1-q+a}, \\
b=\left\|\left(F^{\prime}\left(x_{0}\right)^{T} F^{\prime}\left(x_{0}\right)\right)^{-1} F^{\prime}\left(x_{0}\right)^{T}\right\|,
\end{gathered}
$$

$$
c=\left\|F\left(x_{0}\right)\right\|,
$$

for some $x_{0} \in D$.

We can state the main semilocal convergence theorem for the GaussNewton method (1.3). Let $F: \mathbb{R}^{n} \longrightarrow \mathbb{R}^{m}$ be continuously Fréchetdifferentiable on $U\left(x^{\star}, r_{0}\right)$, for some $x_{0} \in D$ and $r>0$.

Assume:

Operator $F^{\prime}\left(x_{0}\right)$ is of full rank and $F^{\prime}$ satisfies the Lipschitz condition (1.4);

$$
\begin{gathered}
h=b^{2} c \ell=\left\{\begin{array}{lll}
<h_{1} & \text { if } & h_{1} \leq h_{0} \\
\leq h_{0} & \text { if } & h_{0}<h_{1}
\end{array} ;\right. \\
\bar{U}\left(x_{0}, r_{0}\right) \subseteq D,
\end{gathered}
$$

where,

$$
r_{0}=\frac{b c}{1-q} \leq r
$$

Then, sequence $\left\{x_{k}\right\}(k \geq 0)$ generated by the Gauss-Newton method (1.3) is well defined, remains in $\bar{U}\left(x_{0}, r_{0}\right)$ for all $k \geq 0$, and converges to some $x^{\star} \in U\left(x_{0}, r_{0}\right)$.

Moreover, the following estimates hold for all $k \geq 1$ :

$$
\begin{aligned}
\left\|x_{k+1}-x_{k}\right\| & \leq \alpha_{k}\left\|x_{k}-x_{k-1}\right\|^{2}+\beta_{k}\left\|x_{k}-x_{k-1}\right\| \\
q_{k}\left\|x_{k}-x_{k-1}\right\| & \leq q\left\|x_{k}-x_{k-1}\right\|,
\end{aligned}
$$

where,

$$
\alpha_{k}=\frac{b \ell}{2(1-a p h)},
$$


$\beta_{k}=\frac{\sqrt{2} b^{2} \ell}{(1-a p h)\left(1-a p h-b c\left\|x_{k}-x_{k-1}\right\|\right)}\left(c+\frac{\ell}{2} \sum_{i=1}^{i=k}\left\|x_{i}-x_{i-1}\right\|^{2}\right)$,

and

$$
q_{k}=\alpha_{k}\left\|x_{k}-x_{k-1}\right\|+\beta_{k}
$$

Proof of theorem 2. We shall show estimate (2.13), and $x_{k} \in$ $U\left(x_{0}, r_{0}\right)$, for all $k \geq 1$. Note that $x_{1} \in \bar{U}\left(x_{0}, r_{0}\right)$ by the definition of $c$ and (2.12). Using (1.3), (1.5), (2.8)-(2.10), we obtain

$$
\begin{aligned}
\left\|\left(F^{\prime}\left(x_{0}\right)^{T} F^{\prime}\left(x_{0}\right)\right)^{-1} F^{\prime}\left(x_{0}\right)^{T}\right\|\left\|F^{\prime}\left(x_{1}\right)-F^{\prime}\left(x_{0}\right)\right\| & \\
& \leq b \ell_{0}\left\|x_{1}-x_{0}\right\| \\
& \leq b^{2} c \ell_{0} \leq a h<1 .
\end{aligned}
$$

In view of Lemmas 2,2 respectively, and (2.17), we get $F^{\prime}\left(x_{1}\right)$ is of full rank,

$$
\left\|\left(F^{\prime}\left(x_{1}\right)^{T} F^{\prime}\left(x_{1}\right)\right)^{-1} F^{\prime}\left(x_{1}\right)^{T}\right\| \leq \frac{b}{1-a h},
$$

and

$$
\begin{aligned}
\| & \left(F^{\prime}\left(x_{1}\right)^{T} F^{\prime}\left(x_{1}\right)\right)^{-1} F^{\prime}\left(x_{1}\right)^{T}-\left(F^{\prime}\left(x_{0}\right)^{T} F^{\prime}\left(x_{0}\right)\right)^{-1} F^{\prime}\left(x_{0}\right)^{T} \| \\
& \leq \frac{\sqrt{2} b \ell_{0}\left\|x_{1}-x_{0}\right\|}{1-a h} \\
& \leq \frac{\sqrt{2} a h}{1-a h}
\end{aligned}
$$

By (1.3), we obtain the identity

$$
\begin{aligned}
x_{2}-x_{1}= & x_{1}-x_{0}-\left(F^{\prime}\left(x_{1}\right)^{T} F^{\prime}\left(x_{1}\right)\right)^{-1} F^{\prime}\left(x_{1}\right)^{T} F\left(x_{1}\right) \\
& +\left(F^{\prime}\left(x_{0}\right)^{T} F^{\prime}\left(x_{0}\right)\right)^{-1} F^{\prime}\left(x_{0}\right)^{T} F\left(x_{0}\right) \\
= & \left(F^{\prime}\left(x_{0}\right)^{T} F^{\prime}\left(x_{0}\right)\right)^{-1} F^{\prime}\left(x_{0}\right)^{T}\left(F^{\prime}\left(x_{0}\right)\left(x_{1}-x_{0}\right)-F\left(x_{1}\right)+F\left(x_{0}\right)\right)+ \\
& \left.\left(F^{\prime}\left(x_{0}\right)^{T} F^{\prime}\left(x_{0}\right)\right)^{-1} F^{\prime}\left(x_{0}\right)^{T}-\left(F^{\prime}\left(x_{1}\right)^{T} F^{\prime}\left(x_{1}\right)\right)^{-1} F^{\prime}\left(x_{1}\right)^{T}\right) F\left(x_{1}\right) .
\end{aligned}
$$


Using (1.5), we obtain the estimate

$\left\|F\left(x_{1}\right)\right\|$

$\leq\left\|F\left(x_{1}\right)-F\left(x_{0}\right)-F^{\prime}\left(x_{0}\right)\left(x_{1}-x_{0}\right)\right\|+\left\|F\left(x_{0}\right)+F^{\prime}\left(x_{0}\right)\left(x_{1}-x_{0}\right)\right\|$

$\leq \frac{\ell_{0}}{2}\left\|x_{1}-x_{0}\right\|^{2}+\left\|F\left(x_{0}\right)+F^{\prime}\left(x_{0}\right)\left(x_{1}-x_{0}\right)\right\|$

$\leq \frac{b^{2} c^{2} \ell_{0}}{2}+c \leq\left(\frac{a h}{2}+1\right) c$.

By taking norms in (2.20), and using (2.6), (2.18), (2.19), and (2.21), we obtain in turn:

$$
\begin{aligned}
& \left\|x_{2}-x_{1}\right\| \leq\left\|\left(F^{\prime}\left(x_{0}\right)^{T} F^{\prime}\left(x_{0}\right)\right)^{-1} F^{\prime}\left(x_{0}\right)^{T}\right\| \\
& \| \int_{0}^{1}\left(F^{\prime}\left(x_{0}\right)-F^{\prime}\left(x_{0}+\theta\left(x_{1}-x_{0}\right)\right)\left(x_{1}-x_{0}\right) d \theta \|+\right. \\
& \left\|\left(F^{\prime}\left(x_{1}\right)^{T} F^{\prime}\left(x_{1}\right)\right)^{-1} F^{\prime}\left(x_{1}\right)^{T}-\left(F^{\prime}\left(x_{0}\right)^{T} F^{\prime}\left(x_{0}\right)\right)^{-1} F^{\prime}\left(x_{0}\right)^{T}\right\|\left\|F\left(x_{1}\right)\right\| \\
& \leq\left(\frac{b \ell}{2(1-a h)}+\frac{\sqrt{2} a h}{1-a h}\left(\frac{a h}{2}+1\right)\right)\left\|x_{1}-x_{0}\right\| \\
& \leq \alpha_{1}\left\|x_{1}-x_{0}\right\|^{2}+\beta_{1}\left\|x_{1}-x_{0}\right\| \leq q\left\|x_{1}-x_{0}\right\|
\end{aligned}
$$

which shows (2.13) for $k=1$.

We also have

$$
\begin{aligned}
\left\|x_{2}-x_{0}\right\| & \leq\left\|x_{2}-x_{1}\right\|+\left\|x_{1}-x_{0}\right\| \\
& \leq(1+q)\left\|x_{1}-x_{0}\right\|=\frac{1-q^{2}}{1-q}\left\|x_{1}-x_{0}\right\| \leq r_{0}
\end{aligned}
$$

which implies $x_{2} \in \bar{U}\left(x_{0}, r_{0}\right)$.

Let us assume estimate $(2.13)$, and $x_{i} \in \bar{U}\left(x_{0}, r_{0}\right)$ hold true for all $i<k$.

Using the induction hypotheses, (1.5), (2.4), (2.8), and (2.10), we get in turn:

$$
\begin{aligned}
& \left\|\left(F^{\prime}\left(x_{0}\right)^{T} F^{\prime}\left(x_{0}\right)\right)^{-1} F^{\prime}\left(x_{0}\right)^{T}\right\|\left\|F^{\prime}\left(x_{i}\right)-F^{\prime}\left(x_{0}\right)\right\| \\
& \leq b \ell_{0}\left\|x_{i}-x_{0}\right\| \\
& \leq b \ell_{0}\left(\left\|x_{i}-x_{i-1}\right\|+\left\|x_{i-1}-x_{i-2}\right\|+\cdots+\left\|x_{1}-x_{0}\right\|\right) \\
& \leq b \ell_{0}\left(q^{i-1}+q^{i-2}+\cdots+1\right)\left\|x_{1}-x_{0}\right\| \leq a p h<1 .
\end{aligned}
$$

In view of Lemmas 2, 2, and (2.24), we get $F^{\prime}\left(x_{i}\right)$ is of full rank, and

$$
\left\|\left(F^{\prime}\left(x_{i}\right)^{T} F^{\prime}\left(x_{i}\right)\right)^{-1} F^{\prime}\left(x_{i}\right)^{T}\right\| \leq \frac{b}{1-a p h} .
$$


Moreover, we have:

$\left\|\left(F^{\prime}\left(x_{k-1}\right)^{T} F^{\prime}\left(x_{k-1}\right)\right)^{-1} F^{\prime}\left(x_{k-1}\right)^{T}\right\| \ell\left\|x_{k}-x_{k-1}\right\|$

$\frac{\leq}{b \ell q^{n-1}\left\|x_{1}-x_{0}\right\|}$

$\leq$

$\frac{h}{1-a p h}<1$.

Using Lemmas 2, 2, we get

$$
\begin{aligned}
& \left\|\left(F^{\prime}\left(x_{k}\right)^{T} F^{\prime}\left(x_{k}\right)\right)^{-1} F^{\prime}\left(x_{k}\right)^{T}-\left(F^{\prime}\left(x_{k-1}\right)^{T} F^{\prime}\left(x_{k-1}\right)\right)^{-1} F^{\prime}\left(x_{k-1}\right)^{T}\right\| \\
& \leq \frac{\sqrt{2}\left(\frac{b}{1-a p h}\right)^{2} \ell\left\|x_{k}-x_{k-1}\right\|}{1-\frac{b \ell\left\|x_{k}-x_{k-1}\right\|}{a p h}} .
\end{aligned}
$$

By (1.3) as in (2.20), we obtain the identity:

$$
\begin{aligned}
& x_{k+1}-x_{k}= \\
& \left(F^{\prime}\left(x_{k-1}\right)^{T} F^{\prime}\left(x_{k-1}\right)\right)^{-1} F^{\prime}\left(x_{k-1}\right)^{T} \int_{0}^{1}\left(F\left(x_{k-1}\right)-F\left(x_{k-1}+\theta\left(x_{k}-x_{k-1}\right)\right)\right) \\
& \left(x_{k}-x_{k-1}\right) d \theta+\left(F^{\prime}\left(x_{k}\right)^{T} F^{\prime}\left(x_{k}\right)^{-1} F^{\prime}\left(x_{k}\right)^{T}-\left(F^{\prime}\left(x_{k-1}\right)^{T} F^{\prime}\left(x_{k-1}\right)\right)^{-1}\right. \\
& \left.F^{\prime}\left(x_{k-1}\right)^{T}\right) F\left(x_{k}\right) .
\end{aligned}
$$


We also need the estimate

$\left\|F\left(x_{k}\right)\right\| \leq\left\|F\left(x_{k}\right)-F\left(x_{k-1}\right)-F^{\prime}\left(x_{k-1}\right)\left(x_{k}-x_{k-1}\right)\right\|+$

$$
\begin{aligned}
& \left\|F\left(x_{k}\right)+F^{\prime}\left(x_{k-1}\right)\left(x_{k}-x_{k-1}\right)\right\| \\
\leq & \frac{\ell}{2}\left\|x_{k}-x_{k-1}\right\|^{2}+\left\|F\left(x_{k}\right)+F^{\prime}\left(x_{k-1}\right)\left(x_{k}-x_{k-1}\right)\right\| \\
\leq & \left\|F\left(x_{0}\right)\right\|+\frac{\ell}{2} \sum_{i=1}^{i=k}\left\|x_{i}-x_{i-1}\right\|^{2} .
\end{aligned}
$$

By (2.26)-(2.29), we get in turn

$$
\begin{aligned}
& \left\|x_{k+1}-x_{k}\right\| \leq\left\|\left(F^{\prime}\left(x_{k-1}\right)^{T} F^{\prime}\left(x_{k-1}\right)\right)^{-1} F^{\prime}\left(x_{k-1}\right)^{T}\right\| \\
& \| \int_{0}^{1}\left(F^{\prime}\left(x_{k-1}\right)-F^{\prime}\left(x_{k-1}+\theta\left(x_{k}-x_{k-1}\right)\right)\left(x_{k}-x_{k-1}\right) d \theta \|+\right. \\
& \left\|\left(F^{\prime}\left(x_{k}\right)^{T} F^{\prime}\left(x_{k}\right)\right)^{-1} F^{\prime}\left(x_{k}\right)^{T}-\left(F^{\prime}\left(x_{k-1}\right)^{T} F^{\prime}\left(x_{k-1}\right)\right)^{-1} F^{\prime}\left(x_{k-1}\right)^{T}\right\| \\
& \left\|F\left(x_{k}\right)\right\| \\
& \leq \alpha_{k}\left\|x_{k}-x_{k-1}\right\|^{2}+\beta_{k}\left\|x_{k}-x_{k-1}\right\| .
\end{aligned}
$$

We also have by the definition of $q$ and (2.6):

$$
\begin{aligned}
q_{k} \leq & \frac{b^{2} c \ell q^{k-1}}{2(1-a p h)}+\frac{\sqrt{2}\left(\frac{b}{1-a p h}\right)^{2} \ell}{1-\frac{b \ell q^{n-1}\left\|x_{1}-x_{0}\right\|}{1-a p h}} \\
& \left(c+\frac{c h}{2}+\frac{\ell}{2} \sum_{i=1}^{i=k-1} q^{2 i}\left\|x_{1}-x_{0}\right\|^{2}\right) \\
\leq & \frac{h}{2(1-a p h)}+\frac{\sqrt{2} h\left(1+\frac{1}{2} h\left(1+p_{1}\right)\right)}{(1-a p h)(1-a p h-h)} \leq q
\end{aligned}
$$


which completes the induction for (2.13).

Moreover, we have:

$$
\begin{aligned}
\left\|x_{k+1}-x_{0}\right\| \leq & \left\|x_{k+1}-x_{k}\right\|+\left\|x_{k}-x_{k-1}\right\|+\cdots+\left\|x_{1}-x_{0}\right\| \\
\leq & \left(q^{k}+q^{k-1}+\cdots+1\right)\left\|x_{1}-x_{0}\right\| \\
& =\frac{1-q^{k+1}}{1-q}\left\|x_{1}-x_{0}\right\| \leq r_{0},
\end{aligned}
$$

which implies $x_{k+1} \in \bar{U}\left(x_{0}, r_{0}\right)$.

Let $j \geq 0$. We get

$$
\begin{aligned}
\left\|x_{k+j}-x_{k}\right\| \leq & \left\|x_{k+j}-x_{k+j-1}\right\|+\left\|x_{k+j-1}-x_{k+j-2}\right\|+\cdots+\left\|x_{k+1}-x_{k}\right\| \\
\leq & \left(q^{j-1}+q^{j-2}+\cdots+1\right)\left\|x_{k+1}-x_{k}\right\| \\
& =\frac{1-q^{j}}{1-q} q^{k}\left\|x_{1}-x_{0}\right\| \leq r_{0},
\end{aligned}
$$

which by letting $j \longrightarrow \infty$ implies $\lim _{k \rightarrow \infty} x_{k}=x^{\star} \in \bar{U}\left(x_{0}, r_{0}\right)$ (since $\bar{U}\left(x_{0}, r_{0}\right)$ is a closed set), and

$$
\left\|x_{k}-x^{\star}\right\| \leq \frac{q^{k}}{1-q}\left\|x_{1}-x_{0}\right\| .
$$

That completes the proof of Theorem 2 .

$\underline{\text { Case } \ell=\ell_{0}}$

Using (2.1)-(2.7), we get

$$
\begin{aligned}
& q=.363504384, \quad p=1.57110273, \quad p_{1}=1.52126262 \\
& f_{1}(t)=-1.307374992 t^{2}+6.83984613 t-.727008768 \\
& h_{0}=.108542119 \quad \text { and } \quad h_{1}=.388938173 .
\end{aligned}
$$

Condition (2.10) becomes

$$
h \leq .108542119=h_{2} .
$$

The corresponding $\bar{q}, \bar{h}$ given by Li and Zhang in [8] are

$$
\bar{q}=.5>q
$$


and

$$
\bar{h}>h .
$$

Hence, we have expanded with applicability of Gauss-Newton method (1.3) under the same computational cost and with a smaller ratio than in [8].

In the next case, we take advantage of the case $a<1$.

\section{$\underline{\text { Case } a=.5}$}

As in the previous case we obtain

$$
\begin{aligned}
& q=.491020341, \quad p=1.964715057, \quad p_{1}=.31528735, \\
& f_{1}(t)=2.034670476 t^{2}-6.739897921 t+.982040682 \geq 0, \\
& h_{0}=.15274924, \quad h_{1}=.504449871 \quad \text { and } \quad h=h_{0} \leq .15274924=h_{3},
\end{aligned}
$$

which extends further the applicability of Gauss-Newton method, since

$$
h_{3}>h_{2}
$$

where $h_{2}$ is given by $(2.35)$.

Finally, note that if $a \longrightarrow 0^{+}$, we get by $(2.1)-(2.7)$ that $q \longrightarrow .69722436$, $h_{1} \longrightarrow 1$, and $h_{0} \longrightarrow .246590672$. That is the largest possible upper bound on $h$ given in $(2.10)$ is $h_{0}=.246590672$.

The advantages obtained here can also be extended to the case of the Newton-type method

$$
x_{n+1}=x_{n}-F^{\prime}\left(x_{n}\right)^{+} F\left(x_{n}\right) \quad(n \geq 0)
$$

analyzed by Ben-Israel in [4]. However, we leave the details to the motivated reader.

\section{References}

[1] I. K. Argyros, A unifying local-semilocal convergence analysis and applications for two-point Newton-like methods in Banach space, J. Math. Anal. and Appl., 298, pp. 374-397, (2004). 
[2] I. K. Argyros, A convergence analysis of Newton-like methods for singular equations using outer or generalized inverses, Applicationes Mathematicae, 32, pp. 37-49, (2005).

[3] I. K. Argyros, Convergence and applications of Newton-type iterations, Springer Verlag Publ., New York, (2008).

[4] A. Ben-Israel, A Newton-Raphson method for the solution of systems of equations, J. Math. Anal. Appl., 15, pp. 243-252, (1966).

[5] J. M. Gutiérrez, A new semilocal convergence theorem for Newton's method, 79, pp. 131-145, (1997).

[6] Z. Huang, The convergence ball of Newton's method and the uniqueness ball of equations under Hölder continuous derivatives, Comput. Appl. Math., 47, pp. 247-251, (2004).

[7] L. V. Kantorovich, G.P. Akilov, Functional analysis in normed spaces, Pergamon Press, New York, (1982).

[8] C. Li, W. Zhang, Convergence of Gauss-Newton's method, J. of Southeast University, (Don Nan Da Xue Xue Bao), (Natural Science Edition in Chinese), Vol. 31, 5, sept., pp. 135-138, (2001).

[9] P. A. Wedin, Perturbation theory for pseudo-inverse, BIT, 13, pp. 217-232, (1973).

[10] Y. Yuan, W. Sun, Optimization theory and methods. Nonlinear Programming. Springer Optimization and Its Applications, Springer, New York, (2006).

\section{Ioannis K. Argyros}

Department of Mathematics Sciences

Cameron university

Lawton, OK 73505,

U.S.A.

e-mail : iargyros@cameron.edu

and 


\section{Saïd Hilout}

Laboratoire de Mathématiques et Applications

Poitiers university

Bd. Pierre et Marie Curie,

Téléport 2, B.P. 30179

86962 Futuroscope Chasseneuil Cedex,

France

e-mail : said.hilout@math.univ-poitiers.fr 